[WASHINGTON] The US space agency NASA should keep all its current Sun-watching satellites operating until the end of 2001, and should expand the community of researchers analysing their data. That is the conclusion of a panel of experts from outside the agency who had been asked by NASA to review its solar-terrestrial physics programme.

NASA will begin running out of money next year to operate the satellites, and had asked the 'senior review' panel to help it to decide which were most valuable scientifically (see Nature 387, 747; 1997).

But, rather than pick winners and losers, the panel recommended that all eight missions under consideration should have their lives extended. The satellites, which include the European-American Solar and Heliospheric Observatory (SOHO), Ulysses, WIND, POLAR and Voyager, study Sun-Earth interactions from different vantage points in the inner Solar System. They were launched when the Sun was relatively quiet. But now, says the panel, "an opportunity exists, not to be repeated for generations, to use this same array of spacecraft... during the onset and peak of solar maximum conditions".

In fact, says the panel, "it can be argued that the massive [ $\$ 3$ billion] investment in these spacecraft will have been worthwhile only if they are used to provide the same level of information on the important processes of solar maximum", which begins shortly after the turn of the century.

While it found "no redundancy or duplication" among the missions, the panel did say that savings could be achieved in spacecraft operations. And existing scientific teams for the satellites could be pared down by reducing the number of co-investigators. Some 350 scientists are active in the programme, and that number could come down to about 200 in a minimum level 'Solar Maximum Campaign'.

But that will not be enough, says the panel, because understanding the solar maximum is a trickier scientific problem than understanding the quiet Sun. So they "strongly recommend" that NASA should fund an interdisciplinary investigator programme at $\$ 15$ million a year, beginning in 1999, to make up for the shortfall in analytical manpower. Principal investigators would receive additional funds to prepare the satellite data, but the interdisciplinary programme funding would go to support about 125 scientists not directly involved with the project, who would compete for grants.

The panel, chaired by Leonard Fisk of the University of Michigan, a former head of NASA space science, estimates the total funding required for a vigorous Solar Maximum Campaign at \$26 million between 1998 and
2001. "It is at this level, and only this level, that the review panel is prepared to guarantee that an effective program to solve the most complicated, but also the most significant, problems in Sun-Earth-Heliosphere connections will succeed," the panel says.

Whether the group's strong stand will translate into more money for NASA remains to be seen. Guenter Riegler of the agency's Office of Space Science says the recommendations represent "the ideal best of all worlds". Right now, he says, the agency expects to receive only between 60 and 70 per cent of the money it would need to pay for such a programme. The rest presumably would have to come from an additional funding request to Congress beginning with fiscal year 1999.

The review panel acknowledges that the space agency may not be able to afford the programme it recommends. But, rather than start turning off satellites or cutting back on science immediately to make ends meet, the group recommends that NASA should continue a vigorous programme for as long as the moneyholds out.

"An extraordinary opportunity will belost if the full program is not pursued through the expected solar maximum in 2001," the panel says. "However, it would be a greater tragedy not to use fully the available assets while they can be supported."

\title{
Three weeks into mission, Sojourner dusts off Mars' secrets
}

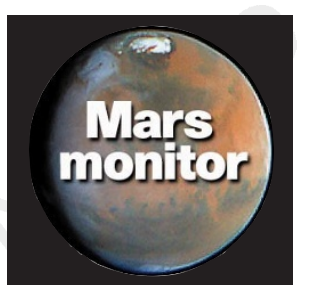

[LONDON] Mars - at least in the small patch of the Ares Vallis being explored by the US Pathfinder mission - is both more varied and more uniform in appearance than expected.

Images from the optical camera on the mission's lander have revealed a relatively colourful landscape, with contrasting reds and greys; yet the elemental compositions of different soils and rocks measured by the Sojourner rover are mostly very similar.

Pathfinder is still operating well, after more than three weeks on the Martian surface. Despite communications problems which have slowed the rover's progress for example, giving it the weekend off on 19 and 20 July - several rocks and soil samples have been analysed using its alpha proton $\mathrm{X}$ ray spectrometer (APXS).

The first rock to be analysed, Barnacle Bill, is the most intriguing so far, being similar in composition to andesites, or basaltic andesites, on Earth. The rock's relatively high silica content suggests it may have been reheated or heated in a watery environment.

In contrast, Yogi - a larger rock that Sojourner took a few days to get to grips with properly - seems to contain lower levels of silica. It may have an ordinary basaltic composition, as expected for most of Mars's crust; but it is covered with dust, which may have made the measurements useless. "Next time, we should take a clothes-brush", comments Rudy Rieder, principal investigator on the APXS.

As a result of this experience, the Pathfinder team decided to clean the next rock. After an initial APXS measurement of the pale-looking Scooby Doo, they rolled the rover back and forth across it, spinning the wheels to try to scrape off as much soil as possible. (Similarly pale material was dug up by Sojourner's wheels on one occasion and may be widespread just below the surface.)

This exercise made little difference to the appearance of Scooby Doo, so it is probably a white rock, rather than a dark one covered in pale dust.

Before the scraping, Scooby Doo appeared to have the same composition as the soils. "That's surprising, as it is a very different colour," says co-investigator Johannes Brückner. "There might be some salt there, but we need more analysis to be able to tell."

The diversity of colour of soil patches may be due to differences in particle size, varying degrees of weathering, or chips of rock scattered in darker soils. Surprisingly, differences in elemental composition do not seem to be responsible.

Martian soil is divided into two grades: fine, bright-red 'drift', with particles smaller than about $40 \mathrm{~mm}$; and coarser, deeper-red 'lag'. Drift is carried around the planet by dust storms, becomes thoroughly mixed on a global scale and therefore probably has an average crustal composition. Lag, on the other hand, had been thought to be of more local origin and thus of varied composition.

But Sojourner has not been able to detect any difference, finding identical compositions to the Viking lander soils in every case. One suggestion is that Lag is compacted drift and the white rocks, such as Scooby Doo, may be compacted dust too, although they must have undergone some kind of chemical or mineralogical change if they are

Stephen Battersby 